\title{
Three Dimensional Measuring Points Locating Algorithm Based Texture-Patched Matrix Completion Algorithm for Indoor 3D REM Design
}

PRADIPTA MAITI ( $\sim$ pradipta.2015dr0295@ece.ism.ac.in )

IITISM: Indian Institute of Technology https://orcid.org/0000-0003-2205-4176

Debjani Mitra

IITISM: Indian Institute of Technology

\section{Research Article}

Keywords: TV-WS, TV-GS, Indoor 3D REM, TPT-MC, TPT-SVT, MPS

Posted Date: September 20th, 2021

DOI: https://doi.org/10.21203/rs.3.rs-761667/v1

License: @ (i) This work is licensed under a Creative Commons Attribution 4.0 International License.

Read Full License 


\title{
Three dimensional measuring points locating algorithm based texture-
}

\section{patched Matrix Completion algorithm for indoor 3D REM design}

\author{
Pradipta Maiti ${ }^{a}$, Debjani Mitra ${ }^{b}$ \\ ${ }^{a}$ Department of Electronics Engineering, IIT(ISM) Dhanbad, Jharkhand, India \\ ${ }^{b}$ Department of Electronics Engineering, IIT(ISM) Dhanbad, Jharkhand, India
}

Abstract - In this paper, a novel texture-patch transformed (TPT) three dimensional (3D) matrix completion (MC) method has been proposed with the support of novel 3D measuring points (MPs) locating algorithm to generate practical received signal strength (RSS) database assisted indoor 3D radio environment map (REM) of ultra-high frequency (UHF) television (TV)-band. The exploration of TV-band results in TV white and grey space (TV-WS and TV-GS), which are competent resolution to recoup excess data traffic through cognitive radio networks (CRNs) by dynamic spectrum access (DSA) by secondary user (SU). Maximum wireless data traffic generates in indoor and altitude considered exploration of REM achieves high data rate, so selecting interpolation algorithm is important for getting accurate and timely generated REM. Many MC algorithm shows better results than standard interpolation methods. Instead of using layer-by-layer MC algorithm, TPT-MC algorithm could be used through 3D↔2D conversion. Patch size has been considered through symmetric dataset profile. MC criteria based analysis shows TPT-MC algorithm takes lesser no. of MPs than layer-by-layer MC algorithm. Singular value thresholding (SVT) algorithm is used MC algorithm. TPT-SVT shows advantage over layer-by-layer SVT algorithm on RMSE, correlation, best-fit-line and simulation time on same no. of dataset. The result analysis shows that TPT-SVT algorithm is better in RMSE, closest best-fit-line and correlation coefficient than 2D IDW2, 2D K-NN, 2D kriging, TPT-IDW2, TPT-KNN, TPT-kriging, 3D IDW2 and layer-by-layer SVT algorithm. Computation time of TPT-SVT is better than 3D IDW2 and SVT. TPT-SVT algorithm takes lesser no. of dataset than SVT algorithm for faithful MC.

Index Terms-TV-WS, TV-GS, Indoor 3D REM, TPT-MC, TPT-SVT, MPs. 


\section{INTRODUCTION}

Cisco visual networking index on global mobile data traffic forecast update of 2017-2022 demonstrates in their white paper that wireless data traffic growth in data traffic has started to exceed the magnitude of spectrum channels for wireless broadband services currently [1]. The advent of Internet of things (IoTs) will further create a new era of traffic overloading and spectrum scarcity [2]. The worldwide "Digital Switch over (DSO) in television (TV) transmission on the other hand has created vacant frequency channels in very high frequency (VHF) and ultra-high frequency (UHF) bands due to their strong penetrating capabilities to provide excellently increased coverage space. The vacant frequency bands are called TV-white space (TV-WS) and partially vacant frequency bands are called TV-grey space (TV-GS). Unlicensed operations in vacant TV band, which are free in space, time and frequency domains, are feasible by dynamic spectrum access (DSA) through cognitive radio networks (CRNs) and it is an active area being researched since past few years [3][4]. The latest IEEE 802.22b standard defines cognitive wireless regional area wifi networks into TV-WS is a new way to address spectrum scarcity, especially important for cost-effective connectivity of portable devices deployed as secondary users in rural area. Pilot projects on TV-WS are being carried out globally to explore the feasibility of commercial success of secondary usage of the unused frequency channels. Many countries has estimated the $\%$ of unused frequency bands in their countries. All 15 channels $(100 \%$ of TV band spectrum) are free in 57\% of the countries and it escalates wireless connectivity requirement [6]. According to National Frequency Allocation Plan (NFAP) - 2018 of India in [7], it has been estimated that Doordarshan has $70-80 \%$ of unused bandwidth or TV-WS in most of its areas. TV-WS could be a better solution for overloaded data of wifi broadcasting [8]. It could be better solution of bandwidth demand for broadband and other services, like- Bluetooth, device to device (D2D) short distance communication, virtual facts, 3D High Definition videos etc. through cognitive access for rapidly increasing overly loaded data traffic.

Practical DSA networks are distinctively supported by geo-location database that stores and delivers the information about location and activity detail of available spectrum bands, interference protection constraints, geographical features, list of frequencies, policies and regulations, received signal strength (RSS) etc. [9]. 
However, there is a need to acquire and process geo-localized sensing and design an exhaustive view of the radio frequency circumstance for coherent dynamic deployment of unused bands. This steers to the concept of radio environment mapping (REM), which makes a smarter geo-location database of multidimensional sharper knowledge thereby imparting better cognitive functionalities to radio nodes [8][10]. REM or spectrum cartography enlightens the status of available radios channels in a given region along with their locations, assigned frequency bands, coverage areas, and interference levels. REM can also be used to construct potential fields for routing and scheduling purpose. REM could be constructed from transmitter database or through field measurement acquired by sensors. Databases generally rely on deterministic or empirical propagation models to construct REM, while spectrum sensing based solutions use actual field measurement and different spatial interpolation technique.

REM construction is commonly approached by direct, indirect or hybrid methods [11][12]. In direct method, sensor based RSS at the sensing nodes using different interpolation techniques generate radio map of a given space. Indirect REM construction method uses the information about propagation pathloss models, transmitter's location and associated parameters. The models being mostly empirical are not very universal and relies on the availability of too many details like - signal transmitting antenna power and height, distance between transmitter and receiver, geographical terrain, altitude of receiver antenna, propagating media density etc. In most of the cases, spectrum sensing is not possible in all altitude varying geo-locations of any continuous attribute that often too irregular to be framed by simple mathematical functions. The variation can be described by a stochastic surface, which could be handled by good spatial estimation techniques. Spatial estimation is to estimate the data at an unobserved point of a spatial phenomenon based on the given data of the phenomenon at observed points. Frequently used direct estimation techniques are inverse distance weight (IDW) and kriging [13]. Their performances in term of computation time, correlation and accuracy cannot be generalized, wherein the nature of dataset plays a strong role in estimation.

Yet another method that has gained popularity in interpolation is matrix completion (MC) technique, in which the aim is to reconstruct all the data of a given matrix using its low or high rank property by using 
small [14]. MC technique has been used to interpolate the missing dataset in various fields, like - seismic data processing, image processing, wireless data processing and others [15]. MC methods has not been exploited much in REM construction in wireless domain. Though MC methods has been used in two dimensional (2D) outdoor scenario, but less explored in indoor three dimensional (3D) special scenario on different frequency bands. TV-WS data are not subjected to sudden changes and have been verified in several studies to have strong spatio-temporal correlation. Singular value thresholding (SVT) is the simplest one [16]. Many other MC algorithms are there [17][18], but the basic criteria for MC are the same.

In wireless communication, $\mathrm{MC}$ algorithms has been used for 2D REM design [19], but still it is untouched in 3D REM design purposes. Few MC algorithms has been used in 3D domain for source localization and sensor placement algorithms based on tensor completion [18][20]. Texture-patch method could be a way of framing the 3D dataset matrix into 2D matrix dataset to fuse the MC algorithms into the framework for 3D data interpolation [19]. Standard interpolation methods need very few scattered dataset for estimation. Most of the MC algorithms are complex and data set specific. MC algorithms has a basic criteria - i) there should be at least one data in each row and column of the matrix and ii) minimum measuring points should be $\geq$ (no. of rows + no. of columns) $\times \operatorname{rank}-\operatorname{rank}^{2}[21]$.

Research works has been performed on 3D dataset interpolation for different purposes using MC through tensor completion in various domains, like - geology, geo-physics, mining, petroleum etc. In wireless domain, few works has been performed on fingerprint based source localization and sensor placement using MC algorithm in 3D domain for wireless local area networks (WLANs) based on tensor completion [22][23]. Most of the sensor placement algorithms has been generated to support standard interpolation algorithms in different fields Very few sensor placement algorithms has been performed to compete with MC algorithms in this fields. In wireless communication field, few sensor placement algorithms has been used been used for wi-fi sensing. Though 2D REM on TV band frequency has been explored in outdoor as well as for indoor using MC algorithms, but 3D REM hasn't been generated using MC algorithms. There is no such sensor 
placement algorithms has been explored to locate the 3D MPs based on texture-patch dimension conversion method.

Recent researches are direct and indirect REM generation models are on 2D outdoor without considering the altitude [12][13][14][24]-[26], but very few works are on indoor 3D environment [20], [27]-[31]. Omnidirectional broadcasting generates 3D REM. 3D REM is generated considering the altitude, whereas no consideration of altitude in 2D REM, which is a big drawback on RSS accuracy in 2D interpolation. Floorto-floor attenuation (FFA) by considering the altitude has a major impact on indoor RSS. On different indoor vertical lines FFA shows a symmetric profile, which is a backbone in generating better indoor 3D REM than 2D on accuracy perspective. Losses due to indoor obstacles, the RSS of active TV channels might go below their threshold values in various indoor locations or it could be said that TV-GS has been identified in various indoor locations. Thus, 3D REM considering the indoor losses shows a better vision to explore TV-GS than 2D REM. 3D REM explores more WSs and GSs than 2D REM has been reported in [20], [28]-[30]. It has been reported in [31] that combined WSs and GSs ratio in 3D indoor is much higher than that from the 2D outdoor. Altitude consideration using FFA explores more TV-GS volume accurately than 2D area for cognitive operations and other short distance applications [18], [20], [27]-[32].

In previous works, it is observed that no indoor 3D REM explored using interpolation methods considering altitude and different indoor losses [28]-[30][33]. Whole spectrum data collected by field measurements considering receiver antenna height $(\mathrm{RAH})$ and there is no exploration of indoor 3D REM by using 3D interpolation [27][20], [31]. As per the author of [27], multi-tier technologies are capable of supporting spatially dense networks, which enables high data rate (DR). High DR require timely and accurate REM for DSA by CRNs. The author has shown that receiver antenna height (RAH) plays a key role on RSS quality and variation of RAH shows good variability of RSS in performance matrix in 3D domain, which could not be captured in 2D domain. As per the author, consideration of RAH variation shows more realistic view of RSS distribution matrix or 3D REM. Result shows that signal-to-interference-plus-noise-ratio 
(SINR) is monastically varying function of RAH. This result motivates to design indoor 3D REM considering the altitude effect.

The main focus of the current work is to establish data sensitive path size based TPT-MC algorithm interpolation algorithm as better in accuracy, correlation and time complexity than standard interpolation algorithms - IDW2, K-NN and Kriging and layer-by-layer MC algorithm on practical dataset in different dimensional mode. Another focus of this paper is to establish a novel 3D MPs locating algorithm for TPT$\mathrm{MC}$ algorithm under the consideration of $\mathrm{MC}$ criteria.

The following are the major contributions of this paper

1. This is the first effort to explore UHF TV band on indoor 3D dataset estimation using a MC algorithm in TPT way. Here, SVT has been used for MC.

2. A novel TPT dimension conversion method based 3D MPs tracking algorithm has been proposed for faithful MC to support any MC algorithm to fuse into 3D dataset.

3. TPT based MC algorithms takes lesser no. of MPs dataset than layer-by-layer MC on the basis of MC criteria.

4. Advantages of TPT-SVT over SVT algorithms through experimental study shows that TPT-SVT shows advantage over SVT algorithm on RMSE, correlation and best fit line among measured and estimated dataset and computation time for the same MPs on 3D dataset estimation.

5. Advantages of TPT-SVT over other standard interpolation methods through result analysis shows that TPT-SVT algorithm performance is better in RMSE, best fit line and correlation coefficient than 2D IDW2, 2D K-NN, 2D kriging and SVT algorithm in a layerd by layerd way.

6. It is seen that TPT-SVT shows better RMSE, best fit line and correlation coefficient than TPT IDW2, TPT K-NN, TPT kriging and 3D IDW2 on 3D dataset interpolation.

7. The TPT-SVT is 2.03 time and 2.3 times faster than 3D IDW2 and SVT algorithm.

8. TPT-SVT based 3D indoor REM has been designed. 
The paper arrangement is given as - Sec.2 literature study of related works, Sec.3 demonstrates standard interpolation algorithms, Sec.4 proposes 3D MPs locating algorithm for faithful TPT-MC and TPT-MC method by considering 3D MPs locating algorithm for 3D REM generation, Sec.5 presents the measurement campaign, Sec.6 demonstrates the results and data analysis and Sec.7 includes the conclusion.

\section{LITERATURE STUDY OF RELATED WORKS}

The author Zhang, Q, Liu, S., Huang, Y. has proposed a novel time-spectrum space 3D-REM in their paper [33]. They have done field measurements for outdoor as well as indoor measurements to detect TV-WS and TV-GS area accurately. Optimal outdoor sensor deployment strategy using channel-location clustering algorithm was designed to construct REM efficiently with minimum sensor nodes. The indoor measurements have done considering altitude to design 3D-REM but there was no use of any MC algorithms or any standard interpolation technologies to interpolate RSS in different indoor locations. There was no mansion of any algorithm for the deployment of minimum sensor nodes. The authors have not considered any outdoor pathloss model accompanied with different indoor losses to make theoretical dataset to establish any 3D interpolation algorithms.

In [18], author's focuses on 3D wireless sensors network (WSNs) design by fingerprint based localization method via tensor completion. The authors have drawn on the spatial correlation of RSS database, explored the low rank property of RSS data matrix and proposed a new training scheme for matrix completion using tensor completion to interpolated missing data on fingerprint database. It is a method for designing 3D REM. In this paper, there is no consideration of indoor losses and best fit pathloss model for database generation. The author of [17] proposed TPT-MC algorithm to reconstruct seismic traces randomly missing from a uniform grid of a 3D seismic volume. They have applied low-rank MC with texture-patch transformation method for 3D seismic data reconstruction. Though this method has been used for seismic data, but it could be applicable to the wireless data also for 3D REM design. 
In [27] variable RAH has been considered instead of fixed RAH to get more realistic view of RSS distribution in 3D domain. Adaptation of this concept results 3D REM. In this paper, variation of signal-tonoise-ratio (SNR) has been shown with respect to RAH, which shows the altitude variation profile of RSS that can explore indoor 3D REM in more details. The author of [29] shows the more communication opportunities of considering 3D spectrum map over 2D spectrum map in indoor and per floor allocation policies. This could explore TV-WS and TV-GS more precisely. This is done by measured RSS, yes no interpolation method has been used. In [30], the authors proved by their experiment that TV-GS can provide additional spectrum in indoor 3D scenario. The indoor RSS scenario for building distance from TV transmitter, losses due to building materials and floor to floor separation effect on RSS has been discussed. These are the supporting parameters to generate indoor 3D REM to explore WSs.

It has been observed in [16] that matrix completion (MC) algorithms, like - singular value thresholding (SVT) based MC algorithm could be used as spatial interpolation algorithm. SVT based MC is more sensitive and efficient to the most of the types of data sets. In wireless communication, it has been used for 2D REM design, still it is unutilized for 3D REM design purposes. Most of the MC algorithms are complex and data set specific. In [18], REM interpolation has been done by sparse recovery technique on wireless local area networks (WLANs) considering a novel grouped sparse based localization scheme for indoor environment. In the paper, there is no consideration of FFA yet, whole process in based upon RSS only. Novel localization scheme has been considered, but the interpolation technique is yet to compare with other standard interpolation techniques for better establishment. As per [34] indoor REMs that will be used for deployment of indoor heterogeneous networks for stable short distance communication using TV-WS. Dynamic deployment scheme of small cells in TV-WS environment has been proposed for getting better throughput in [35]. In [36], some experimental studies were done in indoor same/multi floor environment, validating a previous simulation model and detecting availability of TV-WS and TV-GS in indoor. The experiments are mainly aimed into the detection accuracy of TV-WS area in 2D indoor space for estimating transmitting power of D2D communication using TV-WS in [32]. In [37], 2D outdoor REM has been generated on 
randomly deployed sensors data using kriging, natural neighbor and IDW interpolation methods to identify white spaces inside and in [38], interpolation has done using IDW and Kriging for 2D REM generation. The authors in [39] analyzes the problem of deployment of sensors versus 2D outdoor REM quality in UHF frequency using IDW and kriging interpolation. In these three papers, K-NN hasn't used for interpolation and there is no interpolation of 3D indoor dataset. REM has been generated on UHF band in [40][41] using NN, IDW and Kriging, but there is no exploration done on indoor 3D environment. Authors of [42] address a special interpolation method of convolutional neural networks featuring global and local features for $2 \mathrm{D}$ REM mapping, still 3D exploration is missing. In [43], a proposal has been made by the authors to construct REM based on RSS dataset on special and frequency domain together. Their study shows strong correlation among dataset in frequency domain with existence of pathloss and shadowing. The analysis has done on 2D dataset only and OK method is used for interpolation. No shadowing affected indoor 3D dataset and other interpolation methods are implemented through comparative study yet. In [44], it is seen that K-NN algorithm is being used for fingerprint localization, but K-NN could be broadly used for interpolation based REM generation.

\section{STANDARD 3D INTERPOLATION ALGORITHMS}

The REM is the navigator to DSA in CRNs for secondary user. There are various standard interpolation methods and $\mathrm{MC}$ algorithms are there for the REM generation using small no. of dataset. Inverse distance weight (IDW), kriging, K-nearest neighbor (K-NN) are few standard interpolation algorithms and singular value decomposition algorithm (SVT) is a MC algorithm. Though these algorithms are standard, but still there is some scopes on improvement of the parameters, mainly in accuracy and data correlation point of view by using MC algorithm. SVT is the simplest MC algorithm and it could be used for 3D dataset interpolation through layer-by-layer merged process.

In case of 3D REM design, there is no such direct way to use MC algorithm. So, an existing way of TPT dimension transformed method, novel in communication domain, has been proposed here to cope with SVT based MC algorithm for 3D dataset estimation. 


\section{PROPOSED METHODS}

\subsection{MATHEMATICS BEHIND 3D MP'S LOCATING ALGORITHM FOR FAITHFUL TPT-MC}

In this section, an algorithm has been proposed for minimal 3D random MPs for faithful TPT-MC algorithm. TPT transformation technique is a from $3 \mathrm{D} \leftrightarrow 2 \mathrm{D}$ dimension conversion technique, based on the symmetric profile of the dataset. There is no such MC algorithm exist for 3D interpolation directly. Texturepatch method makes the platform for MC algorithms to fuse into 3D dataset. Though MPs number and location has lesser importance in standard interpolation techniques, but there is some important criteria's in 2D domain for faithful MC - i) there should be at least one data in each row and column of the matrix and ii) the equation for measuring points should be $\geq$ (no. of rows + no. of columns) $\times$ rank - rank $^{2}$. MC criteria and TPT method together makes new criteria for the selection of minimum MPs no. and location of 3D dataset for faithful MC in 2D domain. Patch size should be selected according to the symmetric profile of the dataset.

This 3D MPs locating algorithm is a two-step process: First, 3D data matrix has been converted into 2D data matrix through TPT dimension conversion method and 3D locations has been tracked with their corresponding 2D locations. Secondly, faithful MC criteria for minimal random MPs locating algorithm has been implemented on the 2D data matrix to locate the minimal 2D MPs and its corresponding 3D MPs from first step. Let's take $\mathrm{A}$ is a 3D matrix of length $\mathrm{x}$, width $\mathrm{y}$, height $\mathrm{z}$ and the patch size is taken as $\mathrm{r} \times \mathrm{r} \times \mathrm{z}$, where $r$ is an positive integer no., then $3 \mathrm{D} \rightarrow 2 \mathrm{D}$ TPT mapping will be,

$$
\mathrm{A}^{\mathrm{x}, \mathrm{y}, \mathrm{z}} \stackrel{\mathrm{TPT}}{\longrightarrow} \mathrm{A}^{\mathrm{r}^{2} \mathrm{z}} \frac{x y}{r^{2}}
$$

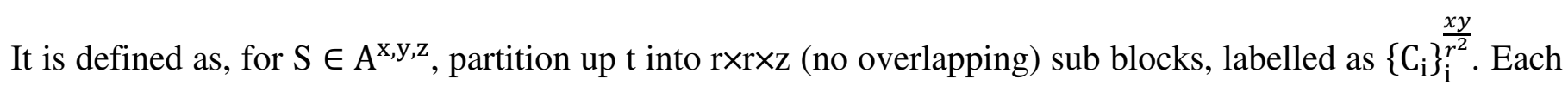
$C_{i}$ is rearranged into a column vector $l_{i}$ of length $r^{2} z$ following the same ordering. Then new transformed matrix will be

TPTt $:=\left[l_{1}, l_{2}, \ldots, \frac{l_{x y}}{\mathrm{r}^{2}}\right]$ 
TPT mapping reduces the nuclear norm of 2D matrices in terms of texture images,

i.e., $\|\mathrm{TPT}(\mathrm{A})\|_{*} \ll\|\mathrm{A}\|_{*}$.

For 3D case, $\|\mathrm{TPTt}\|_{*} \ll\|\mathrm{t}\|_{*}$

$\|\mathrm{t}\|_{*}$ is defined by the trace norm of the tensor. In TPT mapping, a column-by-column raster scan has been performed to arrange the patches into 2D matrix form. The patch size should be set after thorough study of the symmetric profile of the $3 \mathrm{D}$ dataset.

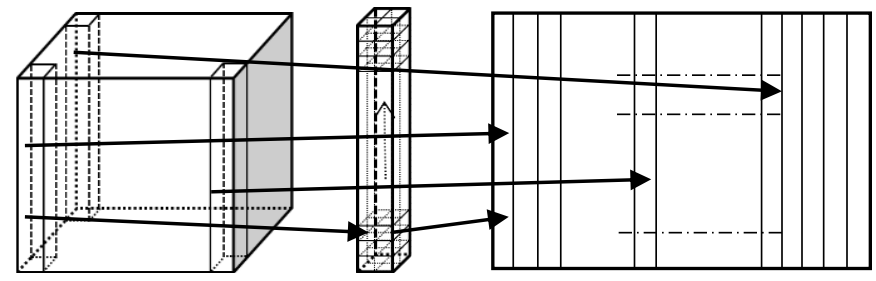

Fig. 1. 3D volume to $2 \mathrm{D}$ surface transform

In this work, the patch size has been set after as $r \times r \times z$ after the through study of the 3D indoor received signal strength (RSS) dataset. As an example, let's take a matrix M of size $4 \times 4 \times 2$. After TPT conversion with patch size of $2 \times 2 \times 2$, the size of the converted matrix $\widetilde{M}$ will be $4 \times 8$.

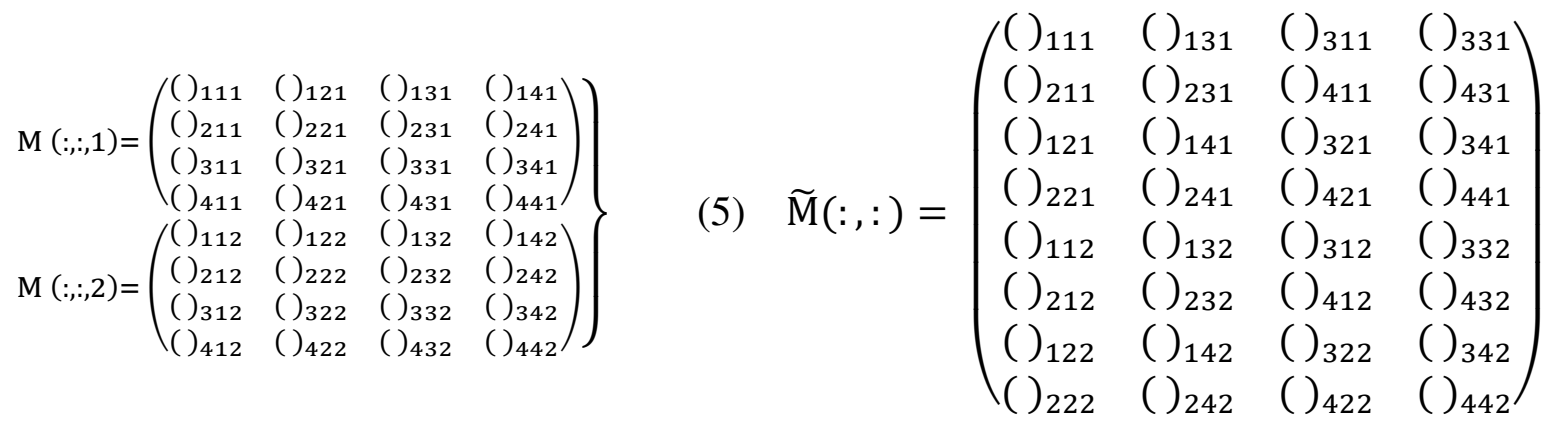

According to the MC criteria - i) there should be at least one data in each row and column of the matrix $\widetilde{M}$ and ii) as per [18] the minimum MPs $m_{p}$ should be

$\mathrm{m}_{\mathrm{p}}>=\left(\mathrm{M}^{\mathrm{W}}+\mathrm{M}^{\mathrm{L}}\right) \times \mathrm{R}-\mathrm{R}^{2}$ 
Where, $M^{W}=\max \left(r^{2} z, \frac{x y}{r^{2}}\right)$ and $M^{L}=\min \left(r^{2} z, \frac{x y}{r^{2}}\right)$ and $R$ is the rank of the matrix $\widetilde{M}$. So, instead of taking 3D measurements randomly, it should be taken in such a way that after $3 \mathrm{D} \rightarrow 2 \mathrm{D}$ TPT conversion given in eqn. (1), there should be at least one data in each row and column of the $2 \mathrm{D}$ matrix $\mathrm{A}^{\mathrm{r}^{2} \mathrm{z}, \frac{\mathrm{xy}}{\mathrm{r}^{2}}}$ and the no. measured data should follow eqn. (7) for faithful MC. Rows and columns may be selected randomly, but the criteria should be the same.

Here, a novel algorithm has been proposed to locate the minimum location no.'s of a 3D matrix $A^{\mathrm{x}, \mathrm{y}, \mathrm{z}}$ for

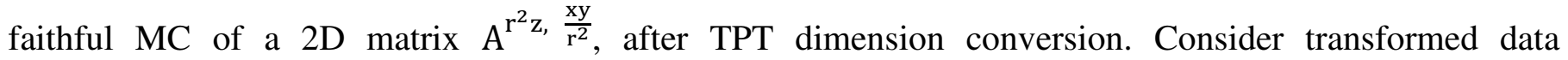

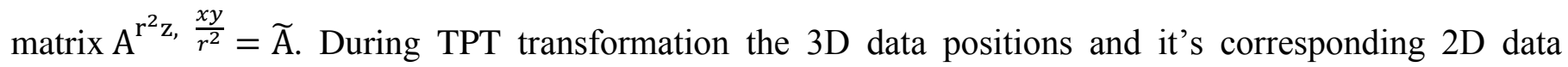
positions has been stored for further position locating for faithful MC. The positional transformation equation will be

$\left.\widetilde{\mathrm{A}}_{\mathrm{X}} \leftarrow \mathrm{f}\left(\mathrm{A}_{\mathrm{X}}^{\mathrm{x}, \mathrm{y}, \mathrm{z}}, \mathrm{A}_{Y}^{\mathrm{x}, \mathrm{y}, \mathrm{z}}, \mathrm{A}_{\mathrm{Z}}^{\mathrm{x}, \mathrm{y}, \mathrm{z}}\right)\right\}$ $\left.\widetilde{A}_{Y} \leftarrow f\left(A_{X}^{\mathrm{x}, \mathrm{y}, \mathrm{z}}, \mathrm{A}_{Y}^{\mathrm{x}, \mathrm{y}, \mathrm{z}}, \mathrm{A}_{\mathrm{Z}}^{\mathrm{x}, \mathrm{y}, \mathrm{z}}\right)\right\}$

Let's take $\mathrm{M}^{\mathrm{W}}$ and $\mathrm{M}^{\mathrm{L}}$ represents the maximum lengths of two dataset, $\mathrm{S}^{\mathrm{M}^{\mathrm{W}}} \subset\left\{1,2, \ldots \ldots, \mathrm{M}^{\mathrm{W}}-1, \mathrm{M}^{\mathrm{W}}\right\}$ and $\mathrm{S}^{\mathrm{M}^{\mathrm{L}}} \subset\left\{1,2, \ldots, \mathrm{M}^{\mathrm{L}}-1, \mathrm{M}^{\mathrm{L}}\right\}$, for storing row and column positions consecutively. Assuming $\mathrm{L}$ and $\mathrm{W}$ are the sets for storing the row and column positions of the selected data positions of the 2D matrix for faithful MC. The row and column positions from datasets $\mathrm{S}^{\mathrm{M}^{\mathrm{W}}}$ and $\mathrm{S}^{\mathrm{M}}$ of the randomly selected $\mathrm{K}^{\text {th }}$ position is represented as $\mathrm{S}_{\mathrm{L}_{\mathrm{K}}}^{\mathrm{M}}$ and $\mathrm{S}_{\mathrm{W}_{\mathrm{K}}}^{\mathrm{M}}$, where $\mathrm{K}=1,2, \ldots, \mathrm{m}_{\mathrm{p}}-1, \mathrm{~m}_{\mathrm{p}}$ and the storing positions will be $\mathrm{L}_{\mathrm{K}}^{\text {th }}$ and $\mathrm{W}_{\mathrm{K}}^{\text {th }}$ of $\mathrm{L}$ and $\mathrm{W}$ sets. The $\mathrm{K}^{\text {th }}$ data of the $2 \mathrm{D}$ matrix for faithful $\mathrm{MC}$ is represented by $\widetilde{\mathrm{A}}_{\left(\mathrm{L}_{\mathrm{K}}, \mathrm{W}_{\mathrm{K}}\right)}$. The conditions to fulfill the first criteria of faithful MC for $\mathrm{K}^{\text {th }}$ position will be

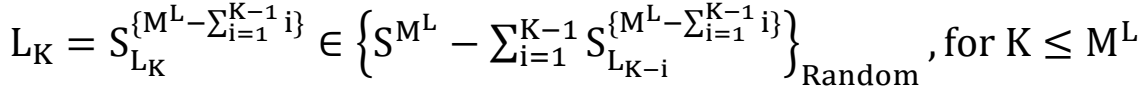

$$
\begin{aligned}
& \mathrm{L}_{\mathrm{K}}=\mathrm{S}_{\mathrm{L}_{\mathrm{K}}}^{\left\{\mathrm{M}^{\mathrm{L}}-\sum_{\mathrm{i}=1}^{\mathrm{K}-1} \mathrm{i}\right\}} \in\left\{\mathrm{S}^{\mathrm{M}^{\mathrm{L}}}\right\}_{\text {Random }}, \text { for } \mathrm{M}^{\mathrm{L}}<\mathrm{K} \leq \mathrm{M}^{\mathrm{W}} \\
& \mathrm{W}_{\mathrm{K}}=\mathrm{S}_{\mathrm{W}_{\mathrm{K}}}^{\left\{\mathrm{M}^{\mathrm{W}}-\sum_{\mathrm{i}=1}^{\mathrm{K}-1} \mathrm{i}\right\}} \in\left\{\mathrm{S}^{\mathrm{M}^{\mathrm{W}}}-\sum_{\mathrm{i}=1}^{\mathrm{K}-1} \mathrm{~S}_{\mathrm{W}-\mathrm{i}}^{\mathrm{W}}\right\}_{\text {Random }}, \text { for } \mathrm{K} \leq \mathrm{M}^{\mathrm{W}}
\end{aligned}
$$


Second criteria of faithful $\mathrm{MC}$ is given in eqn. (7), which necessitates additional minimum $\left(\mathrm{M}^{\mathrm{W}} \times(\mathrm{R}-\right.$ 1) $+M^{L} \times R-R^{2}$ ) dataset. The equations will be $\left.\begin{array}{rl}\mathrm{L}_{\mathrm{K}} & =\left\{\mathrm{S}^{\mathrm{M}^{\mathrm{L}}}\right\}_{\text {Random }} \\ \mathrm{W}_{\mathrm{K}} & =\left\{\mathrm{S}^{\mathrm{M}}\right\}_{\text {Random }}\end{array}\right\}$, for $\mathrm{M}^{\mathrm{W}}<\mathrm{K} \leq \mathrm{m}_{\mathrm{p}} \& \widetilde{\mathrm{A}}\left(\mathrm{L}_{\mathrm{K}}, \mathrm{W}_{\mathrm{K}}\right) \notin\left\{\widetilde{\mathrm{A}}_{\left(\mathrm{L}_{\mathrm{K}-1}, \mathrm{~W}_{\mathrm{K}-1}\right)}, \widetilde{\mathrm{A}}_{\left(\mathrm{L}_{\mathrm{K}-1}, \mathrm{~W}_{\mathrm{K}-1}\right)}, \ldots \ldots, \widetilde{\mathrm{A}}_{\left(\mathrm{L}_{2}, \mathrm{~W}_{2}\right)}, \widetilde{\mathrm{A}}_{\left(\mathrm{L}_{1}, \mathrm{~W}_{1}\right)}\right\}$

The inverse positional transformation equation selects the corresponding $3 \mathrm{D}$ positions of the $2 \mathrm{D}$ positions stored in $\mathrm{L}$ and $\mathrm{W}$ sets. The equations are given below

$\left.\begin{array}{l}A_{X}^{\mathrm{X}, \mathrm{y}, \mathrm{z}} \leftarrow \mathrm{f}\left(\widetilde{\mathrm{A}}_{\mathrm{X}}, \widetilde{\mathrm{A}}_{Y}\right) \\ \mathrm{A}_{Y}^{\mathrm{X}, \mathrm{y}, \mathrm{z}} \leftarrow \mathrm{f}\left(\widetilde{\mathrm{A}}_{\mathrm{X}}, \widetilde{\mathrm{A}}_{\mathrm{Y}}\right) \\ \mathrm{A}_{\mathrm{Z}}^{\mathrm{X}, \mathrm{y}, \mathrm{z}} \leftarrow \mathrm{f}\left(\widetilde{\mathrm{A}}_{\mathrm{X}}, \widetilde{\mathrm{A}}_{\mathrm{Y}}\right)\end{array}\right\}$

\subsection{PROPOSED TPT-MC METHOD BY CONSIDERING 3D MEASURING POINTS LOCATING ALGORITHM FOR 3D REM GENERATION}

In this section, an existing technique has been proposed to solve the problem of using 2D MC algorithms to design indoor 3D REM efficiently in communication field. This will be a novel way to generate 3D models using any MC algorithm [17]. SVT based MC algorithm has been used. In this paper, the proposal has made of a method of $3 \mathrm{D}$ texture-patch transformation that reshapes the $3 \mathrm{D}$ matrix into the $2 \mathrm{D}$, so that $\mathrm{MC}$ algorithms can be used in this framework. The strategy is to cope with the reconstruction of a 3D REM volume. The 3D texture-patch mapping,

TPT: $A^{\mathrm{x}, \mathrm{y}, \mathrm{z}} \rightarrow \mathrm{A}^{\mathrm{r}^{2} \mathrm{z}, \mathrm{xy} / \mathrm{r}^{2}}$

is defined as, for a $S \in A^{x, y, z}$, partition up t into $r \times r \times z$ (no overlapping) sub blocks, labelled as $\left\{\mathrm{C}_{\mathrm{i}}\right\}_{\mathrm{i}}^{\mathrm{xy} / \mathrm{r}^{2}}$. Each $\mathrm{C}_{\mathrm{i}}$ is rearranged into a column vector $\mathrm{l}_{\mathrm{i}}$ of length $\mathrm{r}^{2} \mathrm{z}$ following the same ordering. The newly transformed matrix is given as

$\mathrm{TPTt}:=\left[\mathrm{l}_{1}, \mathrm{l}_{2}, \ldots, \frac{\mathrm{l}_{\mathrm{xy}}}{\mathrm{r}^{2}}\right]$ 
We know TPT mapping reduces the nuclear norm of 2D matrices in terms of texture images,

i.e., $\|\mathrm{TPT}(\mathrm{A})\|_{*} \ll\|\mathrm{A}\|_{*}$.

For a $3 \mathrm{D}$ case, $\|\mathrm{TPT} t\|_{*} \ll\|\mathrm{t}\|_{*}$,

in which the $\|\mathrm{t}\|_{*}$ is defined by the trace norm of the tensor. In this paper, a column-by-column raster scan is used to arrange the patches. Here we take the patch mapping with $2 \times 2 \times 2$ patches applied to a diagonal matrix in (18). The left part is a full-rank diagonal matrix and right part shows a one-rank matrix after transform:

$$
\begin{aligned}
& \left(\begin{array}{llll}
1 & 0 & 0 & 0 \\
0 & 1 & 0 & 0 \\
0 & 0 & 1 & 0 \\
0 & 0 & 0 & 1
\end{array}\right) \Rightarrow\left(\begin{array}{llll}
1 & 0 & 0 & 1 \\
0 & 0 & 0 & 0 \\
0 & 0 & 0 & 0 \\
1 & 0 & 0 & 1
\end{array}\right) \\
& \mathrm{A}(:,:, 1)=\left(\begin{array}{llll}
\mathrm{a}_{111} & \mathrm{a}_{121} & \mathrm{a}_{131} & \mathrm{a}_{141} \\
\mathrm{a}_{211} & \mathrm{a}_{221} & \mathrm{a}_{231} & \mathrm{a}_{241} \\
\mathrm{a}_{311} & \mathrm{a}_{321} & \mathrm{a}_{331} & \mathrm{a}_{341} \\
\mathrm{a}_{411} & \mathrm{a}_{421} & \mathrm{a}_{431} & \mathrm{a}_{441}
\end{array}\right)(19) \mathrm{A}(:,:, 2)=\left(\begin{array}{llll}
\mathrm{a}_{112} & \mathrm{a}_{122} & \mathrm{a}_{132} & \mathrm{a}_{142} \\
\mathrm{a}_{212} & \mathrm{a}_{222} & \mathrm{a}_{232} & \mathrm{a}_{242} \\
\mathrm{a}_{312} & \mathrm{a}_{322} & \mathrm{a}_{332} & \mathrm{a}_{342} \\
\mathrm{a}_{412} & \mathrm{a}_{422} & \mathrm{a}_{432} & \mathrm{a}_{442}
\end{array}\right) \\
& \tilde{A}=\left(\begin{array}{llll}
a_{111} & a_{131} & a_{311} & a_{331} \\
a_{211} & a_{231} & a_{411} & a_{431} \\
a_{121} & a_{141} & a_{321} & a_{341} \\
a_{221} & a_{241} & a_{421} & a_{441} \\
a_{112} & a_{132} & a_{312} & a_{332} \\
a_{212} & a_{232} & a_{412} & a_{432} \\
a_{122} & a_{142} & a_{322} & a_{342} \\
a_{222} & a_{242} & a_{422} & a_{442}
\end{array}\right)
\end{aligned}
$$

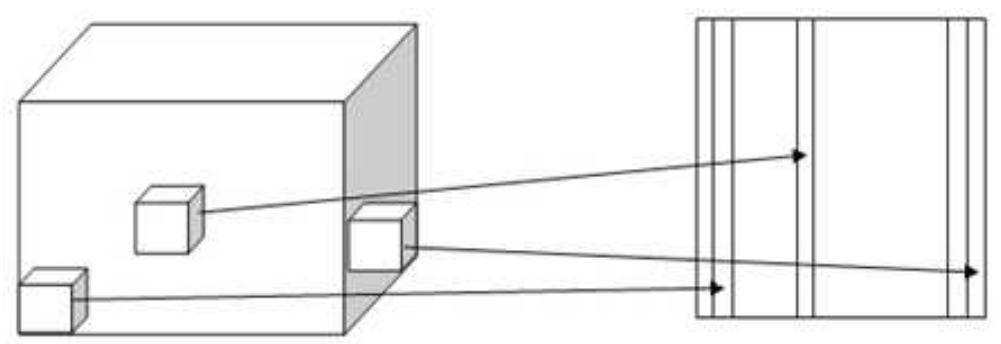

Fig. 2. $3 \mathrm{D}$ volume to $2 \mathrm{D}$ surface transform

Illustration of 3D texture-patch mapping has shown in fig.2. Equation (18) and (19) illustrate more details for the mapping, where we assume a $4 \times 4 \times 2$ data to be mapped into a new matrix of $8 \times 4$ by TPT mapping method. The transformed matrix has shown in eqn.(20). 
Consider $\widetilde{A}$ as transformed data matrices and $\mathrm{a}_{\mathrm{i}, \mathrm{j}}$ are its entries. Each column of $\mathrm{A}$ denotes a trace of spectrum data. $\mathrm{S}(\mathrm{A})$ vector consists singular values of $\widetilde{\mathrm{A}}$ in descending order, $\sigma_{\mathrm{i}}(\widetilde{\mathrm{A}})$ is the $\mathrm{i}^{\text {th }}$ largest singular value and

$\|\widetilde{\mathrm{A}}\|_{\mathrm{F}}=\left(\sum_{\mathrm{i}, \mathrm{j}}\left|\mathrm{a}_{\mathrm{i}, \mathrm{j}}^{2}\right|\right)^{1 / 2}$

is the Frobenius norm of matrix $\widetilde{\mathrm{A}}$. The nuclear norm or trace norm is defined as

$\|\widetilde{\mathrm{A}}\|_{*}=\|\mathrm{S}(\widetilde{\mathrm{A}})\|_{1}=\sum_{\mathrm{i}} \sigma_{\mathrm{i}}(\widetilde{\mathrm{A}})$

The SVT is defined as

$\Gamma_{\tau}(\widetilde{\mathrm{A}})=\mathrm{US}_{\tau} \mathrm{V}^{\mathrm{T}}$

Where, $\mathrm{U}$ and $\mathrm{V}$ denotes left and right matrix of singular value decomposition of $\widetilde{\mathrm{A}}$ and soft thresholding value is taken as $\tau$.

$S_{\tau}=\operatorname{diag}(\max (\sigma-\tau, 0))$

We consider a reconstruction problem of missing traces

$\mathrm{B}=\mathrm{P}_{\Omega}(\widetilde{\mathrm{A}})$

Where $\widetilde{A}$ original spectrum data that we want to reconstruct, $\mathrm{B}$ is observed spectrum data with missing traces and $\mathrm{P}_{\Omega}$ denotes trace sampling,

$\left[\mathrm{P}_{\Omega}(\widetilde{\mathrm{A}})\right]_{i, j}=\left\{\begin{array}{cl}\widetilde{\mathrm{A}}_{i, j}, & (\mathrm{i}, \mathrm{j}) \in \Omega \\ 0, & \text { Otherwise }\end{array}\right.$

$\Omega$ is the index subsets of observation. $\mathrm{P}_{\Omega}$ is the projection on the observation subspace of data with zeros at the locations of missing traces. Assuming the original data $\widetilde{A}$ is low rank. According to the MC theory, randomly missing data can be reconstructed through a rank-minimizing model:

$\min _{X} \operatorname{rank}(\widetilde{A})$, s.t. $\widetilde{A}_{i, j}=B_{i, j}$ for $(i, j) \in \Omega$ 
Here, $\operatorname{rank}(\widetilde{\mathrm{A}})$ is the number of singular values of $\widetilde{\mathrm{A}}$, i.e., $\|S(\widetilde{\mathrm{A}})\|_{0}, T x$ is a transform to reorganize the data into a new matrix with lower rank than $\widetilde{A}$ itself. We first reconstruct $\widetilde{A}$, and then obtain the A by inverse mapping

$\mathrm{A}=\mathrm{TPT}^{-1}(\widetilde{\mathrm{A}})$

\section{MEASUREMENT CAMPAIGN}

In this section the detail demonstration of the measurements campaign accomplished at New Academic Complex (NAC) building of IIT (ISM) Dhanbad and Doordarshan Kentra (DDK) Dhanbad at Dhanbad city in India has given. The latitudes and longitudes of the NAC building and DDK TV broadcasting antennas are $86^{\circ} 26^{\prime} 26.8^{\prime \prime} \mathrm{N}, 23^{\circ} 48^{\prime} 40.9^{\prime \prime} \mathrm{E}$ and $86^{\circ} 28^{\prime} 08.7^{\prime \prime} \mathrm{N}, 23^{\circ} 48^{\prime} 41.2^{\prime \prime} \mathrm{E}$. The two transmitting TV channels are broadcasted by omni-directional slot array type antennas of gain $10 \mathrm{dBi}$ and they are situated at $40 \mathrm{~m}$ height. The maximum permissible transmitting power of the transmitters are $1 \mathrm{~kW}$. RSS has been sensed and collected in Dell Vostro laptop by National Instrument Universal Software Radio Peripheral (NI USRP2922), interfaced with wideband spectrum sensing feature of LABVIEW software. NI USRP 2922, shown in the fig.3a, has the maximum measurable bandwidth of $25 \mathrm{MHz}$ within the frequency range of $400 \mathrm{MHz}-4 \mathrm{GHz}$. The receiver antenna VDR450 has gain of $2 \mathrm{dBi}$. The distance between TV transmitting antenna and NAC building is $2.89 \mathrm{~km}$ and it is measured from google map. Fig. $3 \mathrm{~b} \& 3 \mathrm{c}$ shows the measurements in a classroom in $3^{\text {rd }}$ and $4^{\text {th }}$ floor.
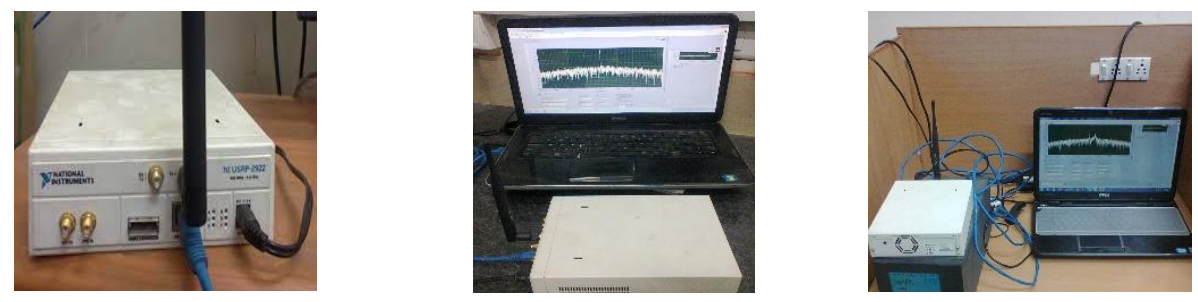

Fig. 3a. NI USRP-2922, Fig. 3b. \& 3c. Measurement setup in a classroom of $3^{\text {rd }}$ and $4^{\text {th }}$ floor of NAC building, IIT(ISM) Dhanbad, 
The authors of [45] demonstrates the frequency range, bandwidth and channel no. of each channel in their paper. The RSS of a channel has been measured at scattered 3D indoor locations in NAC building. The building size is $185 \mathrm{~m} \times 44 \mathrm{~m} \times 36 \mathrm{~m}$ and minimum measurement cube is takes as $5 \mathrm{~m} \times 5 \mathrm{~m} \times 5 \mathrm{~m}$. RSS measurements are collected at each cube multiple times and averaged to eliminate noise. The floor to floor separating distance is $5 \mathrm{~m}$. As it is very time consuming and difficult to measure in all $5 \mathrm{~m} \times 5 \mathrm{~m} \times 5 \mathrm{~m}$ locations several times of the whole building, so few data has been collected and others are interpolated using standard interpolation techniques and MC algorithms. The measurements are taken as per the proposed MPs locating algorithm for faithful MC and few more measurements are taken randomly. Interpolation techniques and data analysis has been performed in MATLAB simulation environment. Few data has been taken from the previous work in [46].

\section{RESULTS AND DATA EVALUATION}

In this section, the analytic results has been shown of the collected data from regourous measurement campaigns in indoor of NAC buliding of IIT(ISM) Dhanbad on 470-590 MHz bandwidth through interfacing USRP2922 and wideband spectrum sensing feature of Labview softwire environment. Total 3040 measurement locations are there of the 3D data matrix of size $38 \times 10 \times 8$. Total $900 \mathrm{RSS}$ data of TV signal of frequency 535.25 MHz has been collected. Comparative study on different standard interpolation algorithms - IDW2, Kriging, K-NN with TPT-SVT algorithm has been performed to see the advantages of using TPTSVT over the standard interpolation algorithms in different dimension and dimension conversion methods from different aspects for 3D dataset interpolation. The advantages of using TPT-SVT over 2D SVT with layer by layer MC has been shown here. RMSE variation in TPT-SVT has shown with respect to variation of different parameters, like - iteration no., delta value and tolerance.

\subsection{Data analysis based on 3D MPs locating algorithm}

In this work, TPT method is a 3D↔2D dimension conversion method, where the patch volume has been selected on the basis of the symmetric characteristics of the dataset. As MC has some criteria on MPs, so, 
TPT based MC or TPT-MC based algorithms should have some criteria for minimum no's of MPs and their scattered 3D locations for faithful MC. There is some advantages of using novel 3D MPs locating algorithm based TPT-MC algorithm in 3D domain instead of using layer by layer 2D MC for missing values estimation of 3D dataset.

Let's take a 3D matrix M of dimension $4 \times 4 \times 2$ and patch size is $2 \times 2 \times 2$. As per the criteria of MC algorithm in eqn. (7), the no. of random MPs for rank $R=1$ of a $8 \times 4$ matrix, should be $\geq(8+4) \times 1-1^{2}=11$ and there should be at least one randomly selected MP in each row and column of the matrix $\widetilde{\mathrm{M}}$. Let's take a rank-1 data matrix which fulfills the two MC criteria

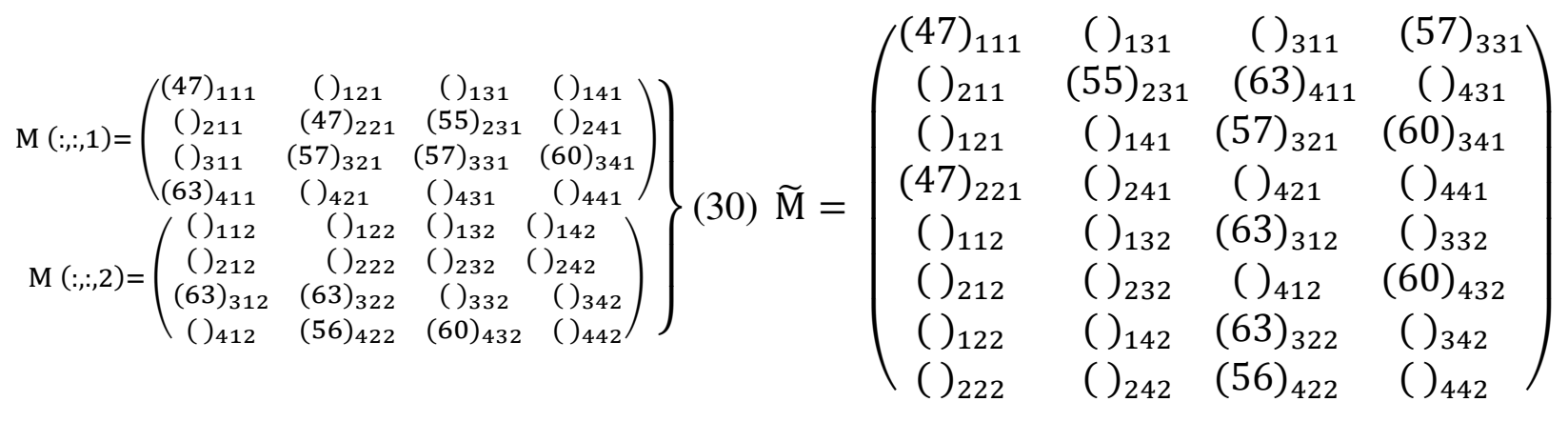

The three digit no. outside each bracket indicates the 3D coordinate of each data location of matrix M. As per MC criteria, $\mathrm{M}(:,:, 1)$ and $\mathrm{M}(:,,, 2)$ each requires at least $(4+4) \times 1-1^{2}=7$, for both $2 \times 7=14$ data for faithful MC, whereas after TPT conversion $\widetilde{\mathrm{M}}$ requires 11 data or lesser no. of data required for matrix $\mathrm{M}$ for faithful MC. In the case of $\widetilde{M}$ first MC criteria has been fulfilled, but it doesn't fulfilled in case of matrix M(:,:,1) and $\mathrm{M}(:,:, 2)$, but it could be fulfilled by reshuffling the dataset as per first MC criteria. Here, singular value decomposition algorithm (SVT) has taken for MC and the result is given below

$$
\widetilde{\mathrm{M}}=\left(\begin{array}{cccc}
(47)_{111} & (46.4)_{131} & (53.2)_{311} & (57)_{331} \\
(55.6)_{211} & (55)_{231} & (63)_{411} & (67.5)_{431} \\
(49.4)_{121} & (48.8)_{141} & (57)_{321} & (60)_{341} \\
(47)_{221} & (46.4)_{241} & (53.2)_{421} & (57)_{441} \\
(55.6)_{112} & (55)_{132} & (63)_{312} & (67.5)_{332} \\
(49.4)_{212} & (48.8)_{232} & (56)_{412} & (60)_{432} \\
(55.6)_{122} & (55)_{142} & (63)_{322} & (67.5)_{342} \\
(49.4)_{222} & (48.8)_{242} & (56)_{422} & (60)_{442}
\end{array}\right)
$$




$$
\begin{aligned}
& \widetilde{\mathrm{M}}=\left(\begin{array}{cccc}
(47)_{111} & (0)_{131} & (53.2)_{311} & (57)_{331} \\
(55.6)_{211} & (0)_{231} & (63)_{411} & (67.5)_{431} \\
(49.4)_{121} & (0)_{141} & (57)_{321} & (60)_{341} \\
(47)_{221} & (0)_{241} & (53.2)_{421} & (57)_{441} \\
(55.6)_{112} & (0)_{132} & (63)_{312} & (67.5)_{332} \\
(49.4)_{212} & (0)_{232} & (56)_{412} & (60)_{432} \\
(55.6)_{122} & (0)_{142} & (63)_{322} & (67.5)_{342} \\
(49.4)_{222} & (0)_{242} & (56)_{422} & (60)_{442}
\end{array}\right) \\
& \widetilde{\mathrm{M}}=\left(\begin{array}{cccc}
(47)_{111} & (46.4)_{131} & (53.2)_{311} & (57)_{331} \\
(55.6)_{211} & (55)_{231} & (63)_{411} & (67.5)_{431} \\
(49.4)_{121} & (48.8)_{141} & (57)_{321} & (60)_{341} \\
(47)_{221} & (46.4)_{241} & (53.2)_{421} & (57)_{441} \\
(55.6)_{112} & (55)_{132} & (63)_{312} & (67.5)_{332} \\
(49.4)_{212} & (48.8)_{232} & (56)_{412} & (60)_{432} \\
(0)_{122} & (0)_{142} & (0)_{322} & (0)_{342} \\
(49.4)_{222} & (48.8)_{242} & (56)_{422} & (60)_{442}
\end{array}\right) \\
& \widetilde{\mathrm{M}}=\left(\begin{array}{cccc}
(47)_{111} & (0)_{131} & (0)_{311} & (0)_{331} \\
(0)_{211} & (55)_{231} & (63)_{411} & (67.5)_{431} \\
(0)_{121} & (48.8)_{141} & (56)_{321} & (60)_{341} \\
(47)_{221} & (0)_{241} & (0)_{421} & (0)_{441} \\
(0)_{112} & (55)_{132} & (63)_{312} & (0)_{332} \\
(0)_{212} & (48.8)_{232} & (56)_{412} & (60)_{432} \\
(0)_{122} & (55)_{142} & (63)_{322} & (67.5)_{342} \\
(0)_{222} & (48.8)_{242} & (56)_{422} & (60)_{442}
\end{array}\right)
\end{aligned}
$$

As both MC criteria has been fulfilled, so faithful MC has been established. It is observed that if any row or column of matrix $\widetilde{\mathrm{M}}$ made vacant completely or no. of MPs reduced to $<11$, then the resultant matrixes are given in eqn.16-18 after SVT based MC will be consecutively. The result shows that if data MP locations doesn't tracked as per the TPT-MC criteria, then after TPT mapping based MC the 3D matrix will not be done properly. It is also observed that TPT-MC based 3D MPs locating algorithm takes lesser no.s of data than normal 2D MC algorithm for faithful MC.

Among 900 measurements there are many measurements are taken at same coordinates with variation in height, among them some are given in the table below. The $\mathrm{x}$, y coordinates of those points are $(36,1),(56$, $1),(81,32)$ and $(127,1)$ and the RSS are measured at each floor. The floor to floor separation is $5 \mathrm{~m}$ and the difference between ground floor and building terrace is $(36-1)=35 \mathrm{~m}$. It is seen from the table- 1 that there 
is a significant variation in the RSS at each coordinate in different floors. These losses are assumed to be due to different indoor losses - doors, windows, wall, partitions and other appliances and reflection. The overall signal attenuation with respect to height of the building has been calculated as a difference between the RSS of ground floor and RSS of terrace at different indoor $(\mathrm{x}, \mathrm{y})$ coordinates. The results shows that with variation of the height $\mathrm{h}$ between outside (terrace) and inside (ground floor) the building, there is a significant symmetric variation of signal or attenuation at the same $(x, y)$ coordinate. In TPT mapping, the value of the height $h$ of the patch size has been set to $\mathrm{r} \times \mathrm{r} \times \mathrm{h}$ by considering this symmetric vertical profile of the dataset.

TABLE 1: FEW RSS AT DIFFERENT FLOORS AND SIGNAL ATTENUATION

\begin{tabular}{|c|c|c|c|c|c|c|}
\hline \multirow[t]{2}{*}{ Floors } & \multirow{2}{*}{$\begin{array}{l}\text { Height } \\
\text { (meter) }\end{array}$} & \multicolumn{5}{|c|}{ Received Signal Strength $(\mathrm{dBm})$ at $(\mathrm{x}, \mathrm{y})$ coordinates } \\
\hline & & $(36,1)$ & $(56,1)$ & $(81,32)$ & $(127,1)$ & $(170,17)$ \\
\hline Ground & 1 & -76.83 & -75.17 & -68.15 & -63.47 & -77.23 \\
\hline $1 \mathrm{st}$ & 6 & -68.14 & -67.33 & -76.5 & -68.42 & -73.15 \\
\hline $2^{\text {nd }}$ & 11 & -66.64 & -53.73 & -71.86 & -69.7 & -69.52 \\
\hline $3^{\text {rd }}$ & 16 & -64.07 & -60.13 & -60.32 & -65.22 & -75.08 \\
\hline $4^{\text {th }}$ & 21 & -70.25 & -56.32 & -56.96 & -64.91 & -73.21 \\
\hline $5^{\text {th }}$ & 26 & -62.56 & -57.37 & -54.87 & -57.08 & -75.49 \\
\hline $6^{\text {th }}$ & 31 & -66.91 & -66.18 & -60.87 & -50.37 & -53.08 \\
\hline Terrace & 36 & -60.5 & -48.67 & -52.81 & -52.48 & -60.93 \\
\hline $\begin{array}{l}\text { Total Attenuation from } \\
\text { terrace to ground floor }\end{array}$ & 35 & 8.69 & 26.5 & 15.33 & 10.98 & 16.3 \\
\hline
\end{tabular}

Another practical example has been taken through implementation of TPT-MC based 3D MPs locating algorithm for the testing and verification of the algorithm in indoor RSS dataset for complete 3D REM generation. In Fig. 4, simulation result of MPs locating algorithm and more measured data points for test 
volume of $16 \mathrm{~m} \times 12 \mathrm{~m} \times 6 \mathrm{~m}$. As $2 \mathrm{~m} \times 2 \mathrm{~m} \times 6 \mathrm{~m}$ patch has taken for the TPT conversion, so the converted matrix will be of size $24 \mathrm{~m} \times 48 \mathrm{~m}$. If the TPT-MC based reconstructed is rank- 2 , then total no. of data required for faithful MC will be $=(24+48) \times 2-2^{2}=140$. Here, 140 data points has been selected according to the MPs locating algorithm and other 181 data points has taken randomly for better accuracy of the interpolated dataset on unmeasured locations. The red dots indicate the MPs according to the MPs locating algorithm and green dots indicate random measuring points in fig.3. If MC has to be done in every z-plane and every reconstructed matrix is assumed to be rank-2, then as per faithful MC no. of data required will be $6 \times\left[(16+12) \times 2-2^{2}\right]=312>140$, which is more than TPT-MC algorithm. TPT-MC based MPs locating algorithm releases the load of excessive and hectic measurements.
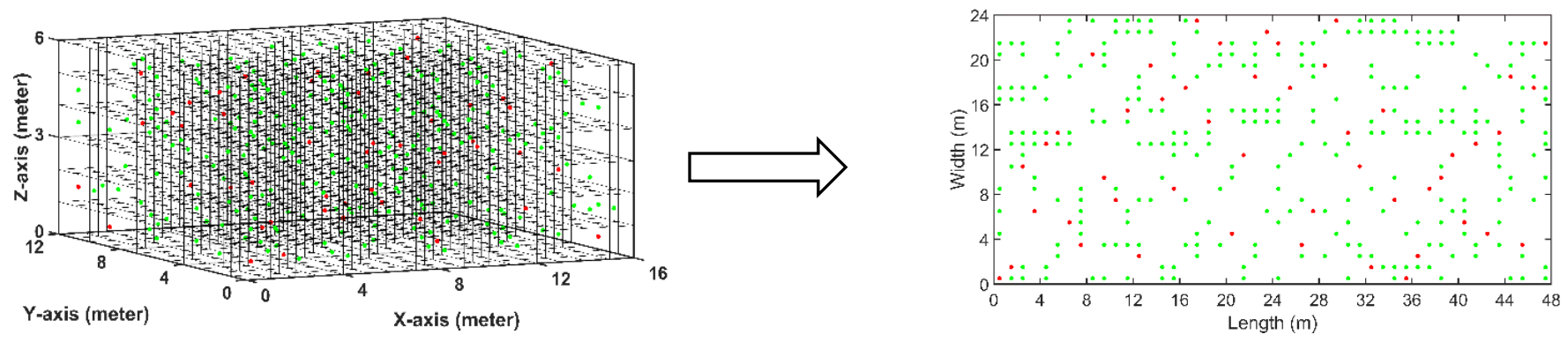

Fig. 4. Measurement locations after $3 \mathrm{D} \rightarrow 2 \mathrm{D}$ conversion

In case of mapping the whole new academic (NAC) building, as it is not possible to measure in all indoor locations, so, TPT-MC based 3D minimum MPs locating algorithm has been implemented on indoor RSS dataset. The whole building has been scaled down to $10 \times 38 \times 8$ matrix, where each unit represents $5 \mathrm{~m}$. After TPT conversion the newly generated matrix is of $32 \times 95$ dimension. If newly generated matrix is rank-2 matrix, then as per the criteria of TPT-MC using minimum MPs locating algorithm the minimum data points required for faithful $\mathrm{MC}$ will be $(32+95) \times 2-2^{2}=250$. Whereas, in case of each z-plane matrix is the matrix is rank-2 matrix, then minimum no. of dataset required for each floor in $10 \times 38$ matrix will be $(10+38) \times 2$ $2^{2}=92$. So, the total data points required for eight floors for faithful $\mathrm{MC}$ will be $=8 \times 92=736$, which is almost three times of the no. of data required for TPT-MC algorithm. It can be conclude from the above two points that TPT based MC is far more advantageous than 2D based MC in case of 3D dataset. As, after TPT 
conversion, the row and column of the newly generated matrix reshuffled, so it may possible that all the row and column of the newly generated matrix may not filled by at least one data. Proposed MPs location tracking algorithm abolishes this problem by randomly selecting the 3D MPs in such a way that after TPT mapping newly generated matrix have at least one data in its each row and column and it follows the criteria of minimum no. of MPs for faithful MC. It has been observed that novel 3D MPs locating algorithm based TPTMC method required lesser no. of dataset, almost $1 / 3$ of the dataset required for layer by layer 2D MC on 3D dataset. It releases the practical problem to collect the practical dataset from a bulk and arduous locations.

\subsection{Comparative study}

Comparison has been done based on two types of parameters - 1) RMSE, 2) Correlation Coefficient and 3) Computation time. The comparative study of interpolated dataset on practical dataset is based upon RMSE and computation time has given in this section. If the no. of testing points are $n$, measured data, interpolated data, mean of the measured data and interpolated data are indicated by $\mathrm{M}, \mathrm{I}, \overline{\mathrm{M}}$ and $\overline{\mathrm{I}}$ consecutively, then equations for RMSE and correlation coefficient will be

$\operatorname{RMSE}=\sqrt{\sum_{\mathrm{i}=1}^{\mathrm{n}} \frac{\left(\mathrm{M}_{\mathrm{i}}-\mathrm{I}_{\mathrm{i}}\right)^{2}}{\mathrm{n}}}$

Correlation Coefficient $=\frac{\sum_{\mathrm{i}=1}^{\mathrm{n}}\left(\mathrm{M}_{\mathrm{i}}-\overline{\mathrm{M}}\right)\left(\mathrm{I}_{\mathrm{i}}-\overline{\mathrm{I}}\right)}{\sqrt{\sum_{\mathrm{i}=1}^{\mathrm{n}}\left(\mathrm{M}_{\mathrm{i}}-\overline{\mathrm{M}}\right) \sum_{\mathrm{i}=1}^{\mathrm{n}}\left(\mathrm{I}_{\mathrm{i}}-\overline{\mathrm{I}}\right)}}$

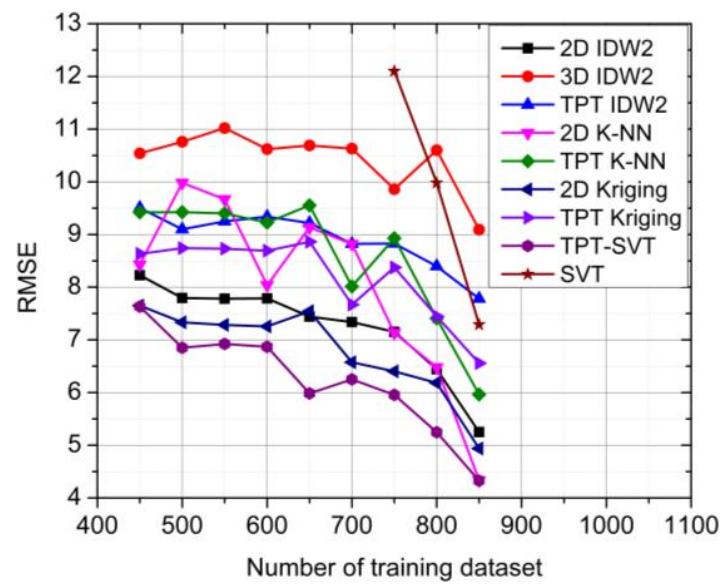

Fig. 5 RMSE vs number of training dataset comparison of different interpolation algorithms 
The pictorial representation of the comparative study on RMSE and correlation coefficient of different interpolation methods and $\mathrm{MC}$ algorithms has been presented in fig.5 and fig.6 consecutively. While comparing on the basis of 2D interpolation, it has been observed that TPT-SVT algorithm shows better RMSE and correlation among measured and interpolate dataset than IDW2, K-NN, kriging and SVT algorithm on 3D dataset interpolation. On the other hand, based on TPT based dimension conversion based interpolation, it has been observed that TPT-SVT algorithm shows better RMSE and correlation among measured and interpolate dataset than IDW2, K-NN, kriging on 3D dataset interpolation. Analysis shows that TPT-SVT performance on RMSE and correlation is better than SVT in layer by layer form for 3D dataset estimation. In case of 3D based interpolation, it has been observed that TPT-SVT algorithm shows better RMSE and correlation among measured and interpolate dataset than IDW2 for 3D dataset interpolation.

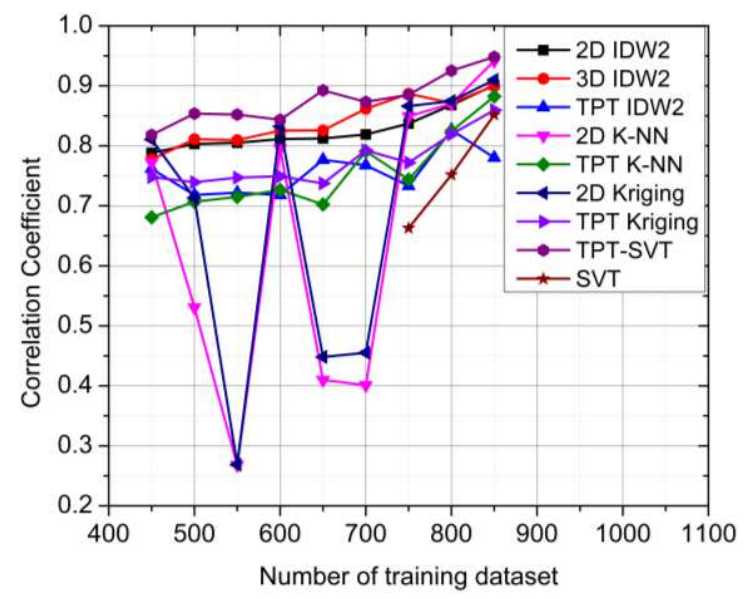

Fig. 6 Correlation coefficient vs number of training dataset comparison of different interpolation algorithms

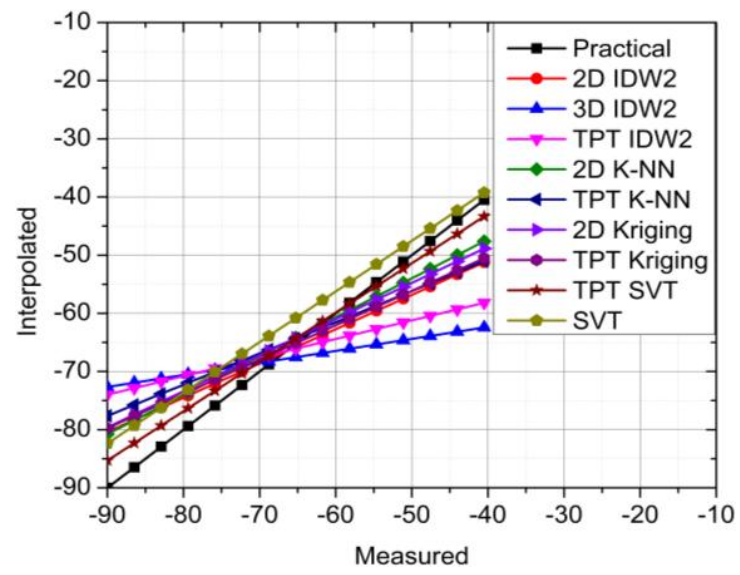

Fig.7 Best fit line analysis between interpolated and measured dataset of different algorithms 
It is observed in fig.7 that TPT-SVT algorithm shows closest best fit line to the best fit line of the practical dataset than other algorithms. In case of simulation time results of the interpolation methods and MC algorithms, it is seen from table-2 that TPT-SVT 2.02 times and 2.3 times faster than 3D IDW2 and SVT algorithm consecutiovely for the same dataset estimation.

TABLE 2: Comparative study on simulation time

\begin{tabular}{|l|l|l|l|}
\hline Interpolation Methods & 3D IDW2 & TPT-SVT & SVT \\
\hline Simulation Time (Sec.) & 42.17 & 20.86 & 48 \\
\hline
\end{tabular}

\subsection{Parameter values and related outcomes of SVT and TPT-SVT for different dataset}

Here, the SVT parameter values delta, threshold value or tau and iteration no. has been shown on different number of training dataset and the resultant values on relative error (RE), relative recovery error (RRE) and relative recovery (RR) in spectral norm has shown in table-3 and table-4 on TPT-SVT and SVT algorithms consecutively.

$\mathrm{RE}=\frac{\operatorname{norm}(\mathrm{b}-\mathrm{X} 1(\mathrm{Omega}))}{\operatorname{norm}(\mathrm{b})} \mathrm{b}=$ test dataset, $\mathrm{X} 1(\mathrm{Omega})=$ estimated dataset, $\mathrm{X} 1=$ recovered matrix, Omega $=$ test locations

$\mathrm{RRE}=\frac{\text { norm }\left(\mathrm{TPTM}-\mathrm{X} 1, \mathrm{fro}^{\prime}\right)}{\text { norm }\left(\mathrm{TPTM}, \mathrm{fro}^{\prime}\right)} ;$ TPTM $=$ Incomplete matrix

$\mathrm{RR}$ in the spectral norm $=\frac{\text { norm }(\mathrm{TPTM}-\mathrm{X} 1)}{\text { norm }(\mathrm{TPTM})}$

In table-3, same parameter values and simulation results has been shown floorwise for SVT based MC algorithm on the same 3D dataset. It has been observed that with the increse in the $\%$ of missing dataset the highest singular value of the incomplete matrix decreases and RE, RRE and RR in spectral norm increases for each floor.In the experimental analysis on the basis of TPT-SVT, the value of delta has taken as constant value 2 . The value of the threhold or tau has been taken as the 100 times of the highest singular value of the incomplete matrix and the interation no. has been taken through empirical testing. The observation of table- 
4 shows that with the increase in the $\%$ of missing dataset the value of the highest singular value and the interation no. decreases for MC, but corresponding RE, RRE and RR in spectral norm increases.

TABLE 3: TPT-SVT PARAMETERS AND OUTPUT

\begin{tabular}{|c|c|c|c|c|c|c|}
\hline$\%$ of Missing Dataset & Delta & Tau & Iteration & $\mathrm{RE}$ & RRE & RR in Spectral Norm \\
\hline 72.04 & \multirow{9}{*}{2} & 115539 & 1168 & 0.056 & 1.65 & 2.44 \\
\hline 73.68 & & 110181 & 1500 & 0.041 & 1.69 & 2.56 \\
\hline 75.33 & & 103466 & 1215 & 0.05 & 1.79 & 2.8 \\
\hline 76.97 & & 99485 & 1206 & 0.05 & 1.83 & 2.96 \\
\hline 78.62 & & 93969 & 930 & 0.06 & 1.91 & 3.18 \\
\hline 80.26 & & 84071 & 849 & 0.057 & 2.04 & 3.63 \\
\hline 81.9 & & 77447 & 700 & 0.066 & 2.15 & 4.02 \\
\hline 83.55 & & 70668 & 648 & 0.066 & 2.27 & 4.44 \\
\hline 85.19 & & 63609 & 658 & 0.06 & 2.4 & 4.98 \\
\hline
\end{tabular}

TABLE 4: LAYER-BY-LAYER SVT PARAMETERS AND OUTPUT

\begin{tabular}{|c|c|c|c|c|c|c|c|}
\hline$\%$ of Missing Dataset & Floor & Delta & Tau & Iteration & $\mathrm{RE}$ & RRE & RR in Spectral Norm \\
\hline \multirow[t]{8}{*}{72.04} & Ground & \multirow{8}{*}{2} & 45030 & 891 & \multirow{8}{*}{0.11} & 1.45 & 2 \\
\hline & $1^{\text {st }}$ & & 43917 & 728 & & 1.8 & 2.61 \\
\hline & $2^{\text {nd }}$ & & 48693 & 327 & & 1.6 & 2.21 \\
\hline & $3^{\text {rd }}$ & & 37282 & 590 & & 1.91 & 2.99 \\
\hline & $4^{\text {th }}$ & & 41342 & 1500 & & 1.57 & 2.18 \\
\hline & $5^{\text {th }}$ & & 38426 & 400 & & 1.58 & 2.17 \\
\hline & $6^{\text {th }}$ & & 36731 & 700 & & 1.48 & 2.09 \\
\hline & Terrace & & 68358 & 2310 & & 0.8 & 0.73 \\
\hline
\end{tabular}




\begin{tabular}{|c|c|c|c|c|c|c|}
\hline \multirow[t]{8}{*}{73.68} & Ground & 41478 & 1200 & \multirow{8}{*}{0.15} & 1.46 & 2.13 \\
\hline & $1^{\mathrm{st}}$ & 41398 & 500 & & 1.82 & 2.73 \\
\hline & $2^{\text {nd }}$ & 46350 & 460 & & 1.63 & 2.32 \\
\hline & $3^{\text {rd }}$ & 35381 & 565 & & 1.94 & 3.13 \\
\hline & $4^{\text {th }}$ & 39144 & 1000 & & 1.6 & 2.28 \\
\hline & $5^{\text {th }}$ & 36018 & 900 & & 1.55 & 2.2 \\
\hline & $6^{\text {th }}$ & 34784 & 700 & & 1.47 & 2.12 \\
\hline & Terrace & 63754 & 1500 & & 0.88 & 0.83 \\
\hline \multirow[t]{8}{*}{75.33} & Ground & 39039 & 700 & \multirow{8}{*}{0.18} & 1.52 & 2.3 \\
\hline & $1^{\mathrm{st}}$ & 39900 & 750 & & 1.98 & 3.01 \\
\hline & $2^{\text {nd }}$ & 42556 & 400 & & 1.62 & 2.4 \\
\hline & $3^{\text {rd }}$ & 33633 & 700 & & 1.98 & 3.26 \\
\hline & $4^{\text {th }}$ & 38239 & 1600 & & 1.63 & 2.34 \\
\hline & $5^{\text {th }}$ & 32595 & 900 & & 1.55 & 2.34 \\
\hline & $6^{\text {th }}$ & 31438 & 113 & & 1.47 & 2.17 \\
\hline & Terrace & 61677 & 1450 & & 0.92 & 0.89 \\
\hline
\end{tabular}

\subsection{RMSE with respect different parameters of TPT-SVT}

In this section, the variation of RMSE with respect to different parameters, like - delta, no. of iterations and tolerance has been shows of the 3D dataset. Various empirical testing shows that in TPT-SVT algorithm, the RMSE value of a dataset varies for different value of delta. In fig.7, the variation of RMSE with respect to delta has shown. It is seen that from 0 to 2 value of delta the RMSE decreases rapidly, at delta $=2$, RMSE becomes minimum and after that with the increase in the value of delta the RMSE starts increasing. Also, it has been observed from logarithmic graph of RMSE with respect to tolerance in fig. 8 that with the decrease in the tolerance value below 0.05, the RMSE of TPT-SVT saturates. Simulation results analysis shown in 
fig.9 that with the increase in the no. of iterations, the RMSE of the TPT-SVT algorithm for different test dataset decreases rapily and saturates.
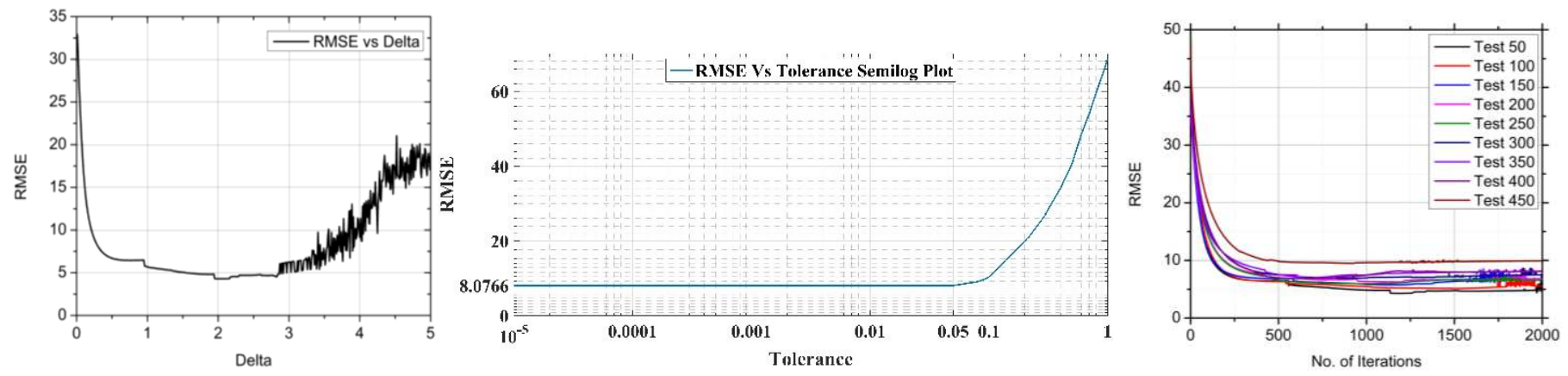

Fig. 7 RMSE vs Delta, Fig. 8 RMSE vs Tolerance, Fig. 9 RMSE vs no. of iterations for different test dataset

In fig.10, 3D indoor REM of a building using TPT-SVT algorithm has shown. The color bar represents the RSS of the indoor dataset in $\mathrm{dBm}$. The threshold value of a TV receiver in $-84 \mathrm{dBm}$ and variation of the color or the RSS explores that there are significant volume of GSs inside the building.

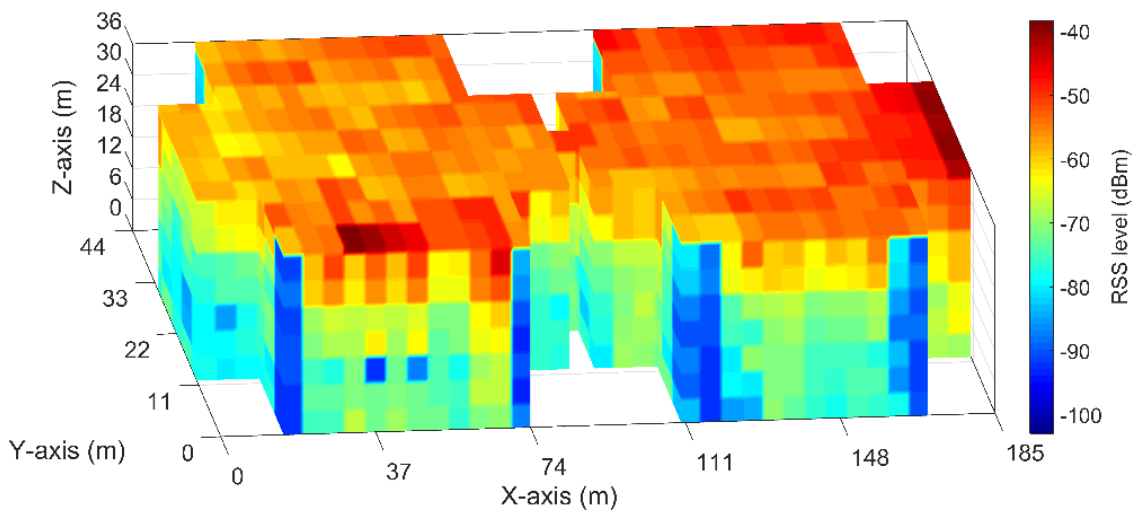

Fig.10 3D indoor REM using TPT-SVT algorithm

\subsection{Cross validation}

The whole experiment has been performed through cross validation results of different test dataset. Total 900 measurements has been divided in different training and test dataset of various combinations of MPs. To get the minimum RMSE and maximum correlation coefficient, best input parameter values, like - tau, delta, no. of iterations and tolerance has been calculated through the study of cross validated results analysis. Also, the outputs parameters, like - RMSE, correlation coefficient, RE, RRE, RR in spectral norm form and simulation 
time has been calculated through cross validation to get the best feasible value for the experimental purposes. The cross validation has been done not only for TPT-SVT and SVT based MC algorithm, also, it has been performed on training datasets for all implemented interpolation methods in this experiment by using in various dimensional platforms.

\section{CONCLUSION}

In this paper, 3D MPs locating algorithm supported TPT-MC algorithm has been proposed and the advantages has been shown through comparative study has been done on different parameters with few standard interpolation algorithms, like-IDW2, KNN and kriging and layer-by-layer SVT algorithm on different dimensional modes, based on practically measured dataset. Practically measured data analysis shows that the patch size is dataset sensitive and it is taken as $\mathrm{r} \times \mathrm{r} \times \mathrm{h}$ after thorough study of the symmetric altitude profile of the dataset. Through study of practically measured dataset shows significant variation or floor to floor signal attenuation of RSS from terrace to the ground floor.

Here, a novel 3D minimum random MPs location tracking algorithm has been proposed to support TPTMC algorithm for faithful MC. Based on the symmetric patch size of the dataset the patch size has been selected for $3 \mathrm{D} \rightarrow 2 \mathrm{D}$ dimension conversion. MC criteria has been considered for $\mathrm{MC}$ on $2 \mathrm{D}$ dataset. As, after TPT mapping the scattered data orientation changes, so, it may possible that newly generated $2 \mathrm{D}$ matrix doesn't fulfill the MC criteria after TPT dimension conversion. TPT-MC criteria based 3D minimum random MPs algorithm solves the problem by selecting the 3D random MPs is such a way that it fulfills the MC criteria after TPT mapping. The 3D random MPs are selected in such a way that after TPT mapping, the 2D matrix should have at least one data in its each row and column and it should have minimum no. of random measuring points $\geq$ (no. of rows + no. of columns $) \times$ rank - rank $^{2}$ for faithful MC. The evaluation of the three test results show that minimum no. of MPs required for TPT-MC based 3D random MPs locating algorithm is much lesser than the data required for layer by layer 2D MC for the any same 3D dataset. This novel 3D random MPs locating algorithm releases the problem of excessive no. of sensing and putting the sensors in 
unreachable difficult positions by reducing the number of measurements and tracking the exact 3D locations before sensing for faithful TPT-MC of 3D dataset.

In this work, the comparative study through empirical testing and verification on parameters, like RMSE, correlation coefficient and simulation time with other standard interpolation methods - IDW2, KNN and kriging and layer-by-layer SVT algorithm in different dimensional modes has been done to see the advantages of TPT-MC algorithm. As per the best of our knowledge, this is the first effort to explore UHF TV band on indoor 3D REM design using MC algorithm in TPT dimension conversion way. The result analysis shows that TPT-SVT algorithm performance is better in RMSE, best fit line analysis and correlation coefficient than 2D IDW2, 2D K-NN, 2D kriging and SVT algorithm in a layerd by layerd way. Also, it is seen that TPT-SVT shows better RMSE, best fit line analysis and correlation coefficient than TPT-IDW2, TPT-K-NN, TPT-kriging and 3D IDW2 on 3D dataset interpolation. The computation time of TPT-SVT shows better performance than 3D IDW2 and SVT algorithm. Another important advantage has seen from the experimental results that TPT-SVT algorithm takes lesser no. of MPs data than SVT algorithm for faithful MC and it shows advantage over SVT algorithm on RMSE, correlation and best fit line analysis among measured and estimated dataset and simulation time for same no. of MPs on 3D dataset estimation. This is the first effort to use MC algorithm in a novel TPT way for indoor 3D REM generation to explore TV-WS and TV-GS of UHF TV band more efficiently for DSA by CRNs for secondary access.

Further study on dataset could be done to set the best patch size for improving accuracy of TPT-MC algorithm. Different MC algorithms and standard interpolation could be used in TPT way to get better results than TPT-SVT algorithm further in future study.

\section{ACKNOWLEDGEMENT}

The work has been supported by Doordarshan Kendra, Dhanbad and Central Public Works Department, Dhanbad. 


\section{Declaration}

Funding: The work is not supported by any types of funding.

Conflicts of interest/Competing interests: We, the authors declare that we have no known competing financial interests or personal relationships that could have appeared to influence the work reported in this paper. No conflict of interest.

Availability of data and material: Data and material will be provided as per request.

Code availability: Nil.

\section{REFERENCES}

[1] Cisco, "Cisco visual networking index (VNI) global mobile data traffic forecast update, 2017-2022 white paper," Ca, Usa, pp. 3-5, 2019, [Online]. Available: http://www.gsma.com/spectrum/wpcontent/uploads/2013/03/Cisco_VNI-global-mobile-data-traffic-forecast-update.pdf.

[2] D. Evans, "The Internet of Things How the Next Evolution of the Internet The Internet of Things How the Next Evolution of the Internet Is Changing Everything,” no. April, 2011.

[3] S. Kawade and M. Nekovee, "Can cognitive radio access to TV white spaces support future home networks?," 2010 IEEE Symp. New Front. Dyn. Spectrum, DySPAN 2010, pp. 0-7, 2010, doi: 10.1109/DYSPAN.2010.5457890.

[4] S. W. Oh, Y. Ma, M. Tao, and E. Peh, "Introduction to Cognitive Radio and Television White Space," 2016.

[5] L. a N. Man, S. Committee, and I. Computer, Part 22: Cognitive Wireless RAN Medium Access Control (MAC) and Physical Layer ( PHY) Specifications : Policies and Procedures for Operation in the TV Bands IEEE Computer Society, vol. 2015, no. July. 2011.

[6] A. Maheshwari, A. Gopalakrishnan, H. A, N. Mangla, P. Bhagavatula, and R. Goyat, "Television White Spaces - Global Developments and Regulatory,” pp. 1-36, 2014. 
[7] R. T. Sataloff, M. M. Johns, and K. M. Kost, "National Frequency Allocation Plan-2018."

[8] X. Zhang, S. Member, and E. W. Knightly, "WATCH : WiFi in Active TV Channels," IEEE Trans. Cogn. Commun. Netw., vol. 2, no. 4, pp. 330-342, 2016, doi: 10.1109/TCCN.2016.2625330.

[9] A. K. Mishra, D. L. Johnson, and E. Challenges, White Space Communication. .

[10] H. Yilmaz Birkan, T. Tugcu, F. Alagöz, and S. Bayhan, "Radio environment map as enabler for practical cognitive radio networks," IEEE Commun. Mag., vol. 51, no. 12, pp. 162-169, 2013, doi: 10.1109/MCOM.2013.6685772.

[11] F. C. (UPC) Ioannis Dagres, Andreas Polydoros (IASA), Janne Riihijärvi, Jad Nasreddine, Petri Mähönen, (RWTH), Liljana Gavrilovska, Vladimir Atanasovski (UKIM), Jaap van de Beek (HWSE), Berna Sayrac, Sebastien Grimoud (FT), Miguel Lopez Benitez, Jordi Perez Romero, R, "Flexible and Spectrum Aware Radio Access through Measurements and Modelling in Cognitive Radio Systems FARAMIR Document Number D4.1 Radio Environmental Maps: Information Models and Reference Model.”

[12] M. Pesko, T. Javornik, A. Košir, M. Štular, and M. Mohorčič, "Radio environment maps: The survey of construction methods," KSII Trans. Internet Inf. Syst., vol. 8, no. 11, pp. 3789-3809, 2014, doi: 10.3837/tiis.2014.11.008.

[13] S. Chaudhari et al., "Spatial Interpolation of Cyclostationary Test Statistics in Cognitive Radio Networks: Methods and Field Measurements," IEEE Trans. Veh. Technol., vol. 67, no. 2, pp. 11131129, 2018, doi: 10.1109/TVT.2017.2717379.

[14] Z. Sabetsarvestani, F. Kiraly, and M. R. D. Rodrigues, "Entry-wise matrix completion from noisy entries," Eur. Signal Process. Conf., vol. 2018-September, pp. 2603-2607, 2018, doi: 10.23919/EUSIPCO.2018.8553561.

[15] B. Recht, “A Simpler Approach to Matrix Completion,” vol. 12, pp. 3413-3430, 2011.

[16] K. Kunisch and B. Vexler, "A SINGULAR VALUE THRESHOLDING ALGORITHM FOR MATRIX COMPLETION,” vol. 46, no. 5, pp. 1726-1753, 2007. 
[17] J. Ma, "Three-dimensional irregular seismic data reconstruction via low-rank matrix completion," Geophysics, vol. 78, no. 5, 2013, doi: 10.1190/GEO2012-0465.1.

[18] S. Lu, J. Zhang, X. Ma, and C. Kan, "Efficient Kernel Based 3-D Source Localization via Tensor Completion," vol. 13, no. 1, pp. 206-222, 2019.

[19] G. Ding, J. Wang, Q. Wu, Y. D. Yao, F. Song, and T. A. Tsiftsis, "Cellular-base-station-assisted device-to-device communications in TV white space," IEEE J. Sel. Areas Commun., vol. 34, no. 1, pp. 107-121, 2016, doi: 10.1109/JSAC.2015.2452532.

[20] I. H. Alshami, N. A. Ahmad, S. Sahibuddin, and F. Firdaus, “Adaptive indoor positioning model based on WLAN-fingerprinting for dynamic and multi-floor environments," Sensors (Switzerland), vol. 17, no. 8,2017 , doi: 10.3390/s17081789.

[21] Z. Xu, "The minimal measurement number for low-rank matrix recovery," Appl. Comput. Harmon. Anal., vol. 44, no. 2, pp. 497-508, 2018, doi: 10.1016/j.acha.2017.01.005.

[22] M. Tang, G. Ding, and S. Member, “A Joint Tensor Completion and Prediction Scheme for MultiDimensional Spectrum Map Construction,” vol. 4, 2016.

[23] S. He and S. H. G. Chan, "Wi-Fi fingerprint-based indoor positioning: Recent advances and comparisons," IEEE Commun. Surv. Tutorials, vol. 18, no. 1, pp. 466-490, 2016, doi: 10.1109/COMST.2015.2464084.

[24] W. Xu, Wu, Daneshmand, Liu, "Location estimation-based radio environment map construction in fading channels," Wirel. Commun. Mob. Comput., no. February 2015, pp. 421-430, 2015, doi: $10.1002 / \mathrm{wcm}$.

[25] M. Pesko, T. Javornik, L. Vidmar, A. Košir, M. Štular, and M. Mohorčič, “The indirect self-tuning method for constructing radio environment map using omnidirectional or directional transmitter antenna," Eurasip J. Wirel. Commun. Netw., vol. 2015, no. 1, pp. 1-12, 2015, doi: 10.1186/s13638015-0297-2.

[26] S. Ulaganathan et al., "Building accurate radio environment maps from multi-fidelity spectrum 
sensing data," Wirel. Networks, vol. 22, no. 8, pp. 2551-2562, 2016, doi: 10.1007/s11276-015-11110.

[27] J. Nasreddine, J. Riihijarvi, A. Achtzehn, and P. Mahonen, "TheWorld is not flat: Wireless communications in 3D environments," 2013 IEEE 14th Int. Symp. a World Wireless, Mob. Multimed. Networks, WoWMoM 2013, 2013, doi: 10.1109/WoWMoM.2013.6583400.

[28] L. Bedogni, M. Di Felice, F. Malabocchia, and L. Bononi, "Indoor communication over TV gray spaces based on spectrum measurements," IEEE Wirel. Commun. Netw. Conf. WCNC, vol. 4, pp. 3218-3223, 2014, doi: 10.1109/WCNC.2014.6953057.

[29] L. Bedogni, A. Trotta, and M. Di Felice, “On 3-dimensional spectrum sharing for TV white and Gray Space networks," Proc. WoWMoM 2015 A World Wirel. Mob. Multimed. Networks, pp. 1-8, 2015, doi: 10.1109/WoWMoM.2015.7158139.

[30] L. Bedogni, F. Malabocchia, M. Di Felice, and L. Bononi, "Indoor Use of Gray and White Spaces: Another Look at Wireless Indoor Communication," IEEE Veh. Technol. Mag., vol. 12, no. 1, pp. 6371, 2017, doi: 10.1109/MVT.2016.2598414.

[31] X. Ying et al., "Exploring indoor white spaces in metropolises," ACM Trans. Intell. Syst. Technol., vol. 9, no. 1, pp. 1-25, 2017, doi: 10.1145/3059149.

[32] S. F. Chou, H. W. Yen, and A. C. Pang, "A REM-enabled diagnostic framework in cellular-based IoT networks," IEEE Internet Things J., vol. 6, no. 3, pp. 5273-5284, 2019, doi: 10.1109/JIOT.2019.2900093.

[33] Q. Zhang, S. Liu, Y. Huang, and Z. Feng, "Time-Spectrum-Space Three Dimensions Radio Environment Map Construction and Utilization in TV White Space,” Wirel. Pers. Commun., vol. 84, no. 4, pp. 2271-2287, 2015, doi: 10.1007/s11277-015-3009-7.

[34] A. Kliks, P. Kryszkiewicz, A. Umbert, J. Pérez-Romero, and F. Casadevall, "TVWS indoor measurements for HetNets," 2014 IEEE Wirel. Commun. Netw. Conf. Work. WCNCW 2014, pp. 7681, 2014, doi: 10.1109/WCNCW.2014.6934864. 
[35] P. Ameigeiras, D. M. Gutierrez-Estevez, and J. Navarro-Ortiz, "Dynamic Deployment of Small Cells in TV White Spaces," IEEE Trans. Veh. Technol., vol. 64, no. 9, pp. 4063-4073, 2015, doi: 10.1109/TVT.2014.2361457.

[36] E. Obregon, L. Shi, J. Ferrer, and J. Zander, "Experimental verification of indoor TV white space opportunity prediction model," 2010 Proc. 5th Int. Conf. Cogn. Radio Oriented Wirel. Networks Commun. CROWNCom 2010, pp. 1-5, 2010, doi: 10.4108/ICST.CROWNCOM2010.9286.

[37] P. L. Ruj, M. Xqdo, and H. G. X. Fr, "Model for Measurement of Radio Environment Maps and location of White Spaces for Cognitive Radio Deployment,” vol. 0, pp. 913-915.

[38] A. Singh Sengar, R. Gangopadhyay, and S. Debnath, "On the construction of radio environment map for underlay device-to-device networks," 2018 24th Asia-Pacific Conf. Commun. APCC 2018, pp. 413-417, 2019, doi: 10.1109/APCC.2018.8633516.

[39] J. Romanik and K. Zubel, "Radio environment maps for military cognitive networks: analysis of measurement-based maps for uhf band,” 2019 Commun. Inf. Technol. Conf. Proceedings, KIT 2019 10th Int. Sci. Conf., pp. 3-8, 2019, doi: 10.23919/KIT.2019.8883500.

[40] M. Suchański, P. Kaniewski, J. Romanik, E. Golan, and K. Zubel, "Radio environment maps for military cognitive networks: density of small-scale sensor network vs. map quality,” Eurasip J. Wirel. Commun. Netw., vol. 2020, no. 1, pp. 1-6, 2020, doi: 10.1186/s13638-020-01803-4.

[41] J. Romanik, E. Golan, K. Zubel, and P. Kaniewski, "Electromagnetic Situational Awareness of Cognitive Radios supported by Radio Environment Maps,” 2019 Signal Process. Symp. SPSympo 2019, pp. 1-6, 2019, doi: 10.1109/SPS.2019.8882065.

[42] R. Hashimoto and K. Suto, "SICNN: Spatial Interpolation with Convolutional Neural Networks for Radio Environment Mapping,” 2020 Int. Conf. Artif. Intell. Inf. Commun. ICAIIC 2020, pp. 167-170, 2020, doi: 10.1109/ICAIIC48513.2020.9065029.

[43] K. Sato, K. Inage, and T. Fujii, "Radio Environment Map Construction with Joint Space-Frequency Interpolation," 2020 Int. Conf. Artif. Intell. Inf. Commun. ICAIIC 2020, pp. 051-054, 2020, doi: 
10.1109/ICAIIC48513.2020.9065217.

[44] X. Fang, Z. Jiang, L. Nan, and L. Chen, "Optimal weighted K-nearest neighbour algorithm for wireless sensor network fingerprint localisation in noisy environment," IET Commun., vol. 12, no. 10, pp. 1171-1177, 2018, doi: 10.1049/iet-com.2017.0515.

[45] M. Deshmukh, K. Patif, F. Frederiksen, K. Skouby, and R. Prasad, "Wireless broadband network on TVWS for rural areas: An Indian perspective," Int. Symp. Wirel. Pers. Multimed. Commun. WPMC, 2013.

[46] P. Maiti and D. Mitra, "Explore TV White Space for indoor small cells deployment and practical pathloss measurement," Proc. 2017 Int. Conf. Innov. Electron. Signal Process. Commun. IESC 2017, pp. 79-84, 2017, doi: 10.1109/IESPC.2017.8071869. 